\title{
Factors associated with future dental care utilization among low-income smokers overdue for dental visits
}

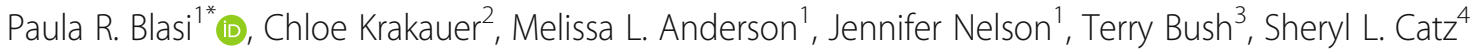
and Jennifer B. McClure ${ }^{1}$

\begin{abstract}
Background: Smokers are at increased risk of oral disease. While routine dental care can help prevent and treat oral health problems, smokers have far lower rates of dental care utilization compared with non-smokers. We sought to better understand which factors may facilitate or hinder dental care utilization among low-income smokers participating in a randomized intervention trial in order to inform future intervention planning.
\end{abstract}

Methods: This is a secondary analysis of data collected between 2015 and 2017 as part of the OralHealth4Life trial. Participants were eligible callers to the Louisiana, Nebraska, and Oregon state tobacco quitlines who had no dental appointment in the prior or upcoming six months. We examined the association between participants' baseline characteristics and their receiving professional dental care between baseline and the 6-month follow-up survey.

Results: Participants were racially diverse (42\% non-White) and two-thirds had an annual household income under $\$ 20,000$. Most (86.7\%) had not had a dental cleaning in more than one year. Commonly cited barriers to dental care included cost (83.7\%) and no dental insurance (78.1\%). Those with dental insurance were more likely to see a dentist at follow-up (RR 1.66). Similarly, those reporting a dental insurance barrier to care were less likely to see a dentist at follow-up (RR 0.69); however, there was no significant utilization difference between those reporting a cost barrier vs. those who did not. After controlling for these financial factors, the following baseline characteristics were significantly associated with a higher likelihood of dental care utilization at 6 months: higher motivation (RR 2.16) and self-efficacy (RR 1.80) to visit the dentist, having a disability (RR 1.63), having a higher education level (RR 1.52), and having perceived gum disease (RR 1.49). Factors significantly associated with a lower likelihood of dental care utilization included being married (RR 0.68) and not having a last dental cleaning within the past year (RR 0.47).

Conclusions: Our findings provide important insight into factors that may facilitate or deter use of professional dental care among low-income smokers. This information could inform the development of future interventions to promote dental care utilization.

Trial registration: ClinicalTrials.gov: NCT02347124; registered 27 January 2015.

Keywords: Oral health, Oral health care, Dental care, Smoking, Tobacco, Dental insurance, Access to dental care, Dental care barriers, Motivation, Self-efficacy

\footnotetext{
* Correspondence: Paula.R.Blasi@kp.org

${ }^{1}$ Kaiser Permanente Washington Health Research Institute, 1730 Minor Ave,

Suite 1600, Seattle, WA 98101, USA

Full list of author information is available at the end of the article
}

(c) The Author(s). 2018 Open Access This article is distributed under the terms of the Creative Commons Attribution 4.0 International License (http://creativecommons.org/licenses/by/4.0/), which permits unrestricted use, distribution, and reproduction in any medium, provided you give appropriate credit to the original author(s) and the source, provide a link to the Creative Commons license, and indicate if changes were made. The Creative Commons Public Domain Dedication waiver (http://creativecommons.org/publicdomain/zero/1.0/) applies to the data made available in this article, unless otherwise stated. 


\section{Background}

Oral health is an important component of overall health and well-being [1]. However, many population groups face a disproportionate burden of oral health problems. Low-income individuals, those with limited education, non-White racial and ethnic groups, and smokers all face higher rates of oral disease than the general population [1-4]. Smokers are a particularly high-risk group because tobacco use is a risk factor for periodontal disease, tooth loss, and oral and pharyngeal cancers [1]. Moreover, smokers are about twice as likely as non-smokers to have not seen a dentist in more than 5 years or to have never seen one $[5,6]$.

Promoting and facilitating regular dental visits is one strategy to address oral health disparities, as routine dental care can help prevent and treat oral disease $[7,8]$. For smokers, routine dental visits may even lessen the negative effects of smoking on oral health because dental providers can evaluate smokers' oral health, check for early signs of oral and pharyngeal cancers, [6] clean their teeth, and counsel them about oral hygiene behaviors such as brushing and flossing [9]. However, despite the potential benefits of dental visits for smokers $[6,9]$, there is little research on best practices for improving dental care utilization among this high-risk population.

To address this knowledge gap, we sought to promote dental care utilization among smokers calling state-funded tobacco quitlines. State quitlines offer free phone-based counseling and other assistance to support callers in quitting smoking [10]. Quitline callers include many populations facing oral health disparities, including low-income individuals, those with limited education, and racial or ethnic groups known to have higher rates of oral disease and lower oral cancer survival rates [11, 12]. Quitline callers also have low rates of dental care utilization, [13] and both quitline callers and quitline providers support the idea of integrating oral health promotion content into quitlines $[10,13,14]$. In addition, smokers who call tobacco quitlines presumably are already interested in behavior change, and therefore may be receptive to interventions to promote dental care.

Together with the quitlines and a team of behavioral scientists and oral health experts, we designed an intervention (OralHealth4Life, or OH4L) to encourage dental care utilization among smokers who are overdue for dental visits. The intervention included oral health counseling from quitline coaches, referral information for local low-cost dental providers, and other motivational and educational content. However, the OH4L intervention did not increase dental care utilization compared to usual quitline care in a randomized controlled trial. About 18\% of both the intervention and control groups reported seeing a dentist during the 6 months following study enrollment [15]. This finding suggests that smokers, particularly those who are more socio-economically disadvantaged and have multiple barriers to care, may require a different type of intervention to increase their use of professional dental care.

There is little existing research on predictors of future dental visits among low-income smokers or on strategies for increasing dental care utilization among low-income smokers who are overdue for dental care. According to Andersen's Behavioral Model of Health Services Use, predictors of health care utilization include predisposing factors (such as demographic characteristics and health beliefs), enabling factors (such as financial resources and access to health insurance), and the need for health services [16, 17]. Thus, the goal of the current analysis was to better understand which potential predisposing and enabling factors may facilitate or hinder future dental care utilization among smokers who are overdue for dental care. Based on prior research, we anticipated that lacking dental insurance, self-reporting dental insurance as a barrier, and self-reporting cost as a barrier would be associated with a lower likelihood of seeing a dentist, as previous research shows that a lack of dental insurance and cost are the most commonly cited reasons for forgoing needed dental care [5, 18-20]. Therefore, a primary aim of this analysis was to assess the influence of additional factors on dental care utilization, after accounting for these financial barriers. Based on prior research, we hypothesized that individuals with lower incomes, those with lower education levels, and those living in rural areas would be less likely to see a dentist [6, 21-23]. Based on common health promotion theories and supporting research, we also hypothesized that individuals with higher levels of motivation and self-efficacy for seeing a dentist would be more likely to do so [24-29]. Ultimately, this work seeks to provide important insight into which smokers are more or less likely to see dental care providers, which in turn, could help public health professionals and policymakers design more effective interventions for these individuals in the future.

\section{Methods \\ Overview}

This is a secondary analysis of data collected between June 2015 and March 2017 as part of the OH4L trial. Since the main trial did not find a significant treatment effect, we combined intervention groups for this cohort data analysis. Additional trial details can be found in the published protocol, [30] at ClinicalTrials.gov (NCT02347124), and in the results from 
the main trial [15]. The Kaiser Permanente Washington Institutional Review Board approved this study.

\section{Participants}

Callers to the Oregon (OR), Nebraska (NE), and Louisiana (LA) state tobacco quitlines were invited to be screened for eligibility following registration for services. Callers were eligible to be screened if they were age 18 or older, could read and speak in English, smoked at least 5 cigarettes daily, were ready to quit smoking, and were eligible for their state's multi-call quitline program. To be eligible for the study, participants also had to have at least some of their natural teeth, no dental appointment in the prior or upcoming 6 months, an interest in improving their oral health, access to the internet, and the ability to receive text messages. We excluded individuals who were incarcerated, were receiving inpatient substance abuse treatment, had significant cognitive impairment or psychosis, were unable to read small text, had plans to move in the next 6 months, or had a household member enrolled in the study.

\section{Consent, enrollment, and baseline data collection}

Eligible callers provided verbal consent, completed the baseline assessment, and were randomized to the control or intervention arm. Randomization was stratified by participants' dental insurance (yes vs. no/unsure) and quitline (LA, NE, or OR).

\section{Intervention}

Intervention details are available in the published protocol [30]. Briefly, the control group received the standard quitline program, which included 4 to 5 calls with a quitline coach and mailed and online smoking cessation content. Controls also received an attention-matched text messaging intervention focused on generic health promotion tips, excluding smoking cessation or oral health. The experimental group received the standard quitline program plus additional scripted oral health counseling during each quitline call, mailed and online oral health promotion materials, and text messages focused on oral health promotion. The oral health counseling and materials discussed the benefits of oral health care and provided referral information for local low-cost dental providers. To prevent treatment contamination, different quitline counselors treated control and experimental participants. Finally, experimental participants each received a toothbrush, dental floss, and xylitol gum.

\section{Assessment measures}

Demographic data included age, gender, race/ethnicity, state of residence (LA, NE or OR), education, income, disability status, and marital status (single vs. married or living as married). Geographic classification was assessed using the U.S. Department of Agriculture's rural-urban commuting area (RUCA) codes, [31] based on participant street address. We followed previously used definitions [32] of "urban" as RUCA code 1, "suburban" as RUCA codes 2-6, and "rural" as RUCA codes 7-10.

Oral health-related assessments were self-reported and included standardized items from the 2011-2012 National Health and Nutrition Examination Survey (NHANES), [33] the 2008 National Health Interview Survey (NHIS), [34] and the 2008 Behavioral Risk Factor Surveillance System (BRFSS) [35]. Participants reported whether they thought they had gum disease (yes/no) after being informed that symptoms of gum disease included loose teeth or swollen, receding, sore, or infected gums. Responses of "I don't know" were coded as not having gum disease. Dental insurance status was assessed by asking participants whether they had dental insurance (yes/no, with responses of "I don't know" coded as not having dental insurance). Participants also reported length of time since their last dental cleaning (ranging from "in the last 6 months" to "never" on a six-point Likert scale.)

In addition, perceived barriers to dental care were assessed by asking participants to rate the extent to which they believed each of 11 common barriers - such as cost or no dental insurance - affected their ability to seek dental care. Participants rated each barrier on a five-point Likert scale ranging from 1 ("definitely not true") to 5 ("definitely true") to indicate the extent to which they perceived that factor as affecting their ability to see a dentist. For our analysis and for ease of interpretation, we grouped similar barriers into the following categories: access (unable to find a dentist, too difficult to get to the clinic), psychological (fear or nervousness, dislike going to the dentist), and prioritization (mean to go but put it off, other health concerns are more important, have no problems with teeth or gums, don't have time, do not think of it). We reported the cost barrier and no dental insurance barrier separately, as existing literature suggests these barriers affect rates of dental care utilization $[5,18-20]$. If a participant rated any individual barrier or barrier in a category as present (rating of 4 or 5), we deemed that individual barrier or category of barrier to be present for that participant. It was possible for participants to report cost or a lack of dental insurance as perceived barriers to dental care, even if they also reported having dental insurance. This situation might arise if a participant had dental insurance that covered few services or involved high premiums and copayments.

Participants rated their motivation and self-efficacy to see a dentist in the next 6 months on a five-point Likert scale ranging from 1 ("not at all motivated/confident") to 5 ("very motivated/confident"). We deemed ratings of 
4 and 5 as "high" motivation or self-efficacy and ratings of 3 or lower as "low" motivation or self-efficacy.

For this analysis, the primary outcome measure was receipt of professional dental care between study enrollment and the 6-month follow-up survey. Receipt of professional dental care was self-reported using an item from the 2012 NHIS, which assessed time since last seeing a dentist, orthodontist, oral surgeon, or dental hygienist [36]. We defined utilization as reporting a dental visit in the prior 6 months at the 2-month or 6 -month follow-up survey. Since we excluded individuals from the study who had seen a dentist in the prior 6 months at enrollment, any dental visits reported during the study period represent post-enrollment care. To encourage accurate reporting, we used a modified bogus pipeline methodology [30] which asked participants to provide the contact details for the dental provider they visited. Participants were aware they would be asked for this information if they reported seeing a dental provider during the study period. More than $90 \%$ of participants who reported seeing a dentist at 6 months provided contact information for their provider [15].

\section{Analysis}

In the original randomized trial, dental care utilization did not differ significantly by treatment arm at 6-month follow-up, [15] thus we pooled all participants in the current analysis. We used descriptive statistics to characterize the demographic and oral health characteristics of study participants and their baseline barriers to seeing a dental provider.

Our primary analytic goal was to explore the effects of multiple baseline characteristics of interest (e.g., motivation to see a dentist and timing of last dental cleaning) on dental care utilization after accounting for a priori identified financial variables (dental insurance status, dental insurance barrier, cost barrier) and important potential confounders (age, gender, and state) of these associations. As a preliminary step to first understand the influence of each financial barrier previously shown to be associated with dental care utilization (dental insurance status, a dental insurance barrier, and a cost barrier), [5, 18-20] we estimated the relative risk (RR) of dental care receipt within 6 months of follow-up (yes/no) using a separate, unadjusted loglinear regression model with robust standard errors for each financial factor (i.e., using single variable adjusted models). We used this same approach to assess the unadjusted association between receipt of dental care and each potential demographic confounder (age, gender, and state).

Then, to assess the independent effect of each baseline characteristic on future dental care utilization above-and-beyond these financial factors and potential confounders, we used a 2-step process. In step 1, we calculated the RR of seeing a dentist at any time between baseline and the 6-month follow-up for each baseline characteristic of interest using a separate loglinear regression model with robust standard errors. Each model was adjusted for the a priori financial and demographic factors described above (dental insurance status, dental insurance barrier, cost barrier, age, gender, and state). Analyses were limited to participants providing baseline data for each item of interest.

In step 2, we fit one multivariable adjusted loglinear regression model with robust standard errors that included the a priori financial and demographic factors plus all the baseline characteristics found in step 1 to be associated with dental care utilization at the $p \leq 0.10$ level.

We report $95 \%$ confidence intervals for each RR estimate and $p$-values for Wald tests. All confidence intervals and tests use robust standard errors and all analytic results were produced using the geepack package in $\mathrm{R}$ [37-39].

As a sensitivity analysis, we repeated both step 1 and step 2, limiting the analytic sample to individuals who had not had a dental cleaning in the past year at baseline. The rationale for this analysis was that this subgroup may be more hesitant to seek dental care, and therefore may have different baseline characteristics associated with seeking care.

\section{Results}

\section{Baseline demographic characteristics}

Our final analytic sample consisted of 718 participants from Louisiana (73\%), Nebraska (13\%) and Oregon (13\%) (Table 1). Most participants (59.7\%) smoked 21 or more cigarettes per day. Nearly two-thirds had an annual household income under $\$ 20,000$, and $55.0 \%$ had a high school education or less. Participants were racially diverse $(58.4 \%$ White, $29.1 \%$ Black, and $12.5 \%$ other or multiple races), and most (87.6\%) lived in an urban or suburban area. Nearly all participants were younger than age 65 (mean age 44.3; standard deviation 12.2), and $61.8 \%$ were female. Our sample was representative of typical state quitline callers, with the exception that a higher proportion of study participants were non-White [40]. Baseline characteristics by state are presented in Table 1.

\section{Baseline oral health-related characteristics and self- reported barriers}

At baseline, most participants (86.8\%) had not had a dental cleaning in more than 1 year (Table 1) and $41.7 \%$ had not had one in more than 5 years or had never had one (data not shown). Most participants reported high levels of motivation (81.3\%) and self-efficacy (67.0\%) for visiting 
Table 1 Baseline demographic characteristics of tobacco quitline callers in $\mathrm{OH} 4 \mathrm{~L}$ study by state of residence, $n=718$

\begin{tabular}{|c|c|c|c|c|c|}
\hline & & $\begin{array}{l}\text { Louisiana } \\
N=527 \\
N(\%)\end{array}$ & $\begin{array}{l}\text { Nebraska } \\
N=95 \\
N(\%)\end{array}$ & $\begin{array}{l}\text { Oregon } \\
N=96 \\
N(\%)\end{array}$ & $\begin{array}{l}\text { Overall } \\
N=718 \\
N(\%)\end{array}$ \\
\hline \multirow[t]{3}{*}{ Age categories } & $18-44$ & $237(45.1)$ & $45(48.4)$ & $53(55.2)$ & $335(46.9)$ \\
\hline & $45-64$ & $271(51.5)$ & $45(48.4)$ & $35(36.5)$ & $351(49.1)$ \\
\hline & 65 or older & $18(3.4)$ & $3(3.2)$ & $8(8.3)$ & $29(4.1)$ \\
\hline \multirow[t]{3}{*}{ Cigarettes per day } & 10 or few & $51(9.7)$ & $9(9.5)$ & $10(10.5)$ & $70(9.8)$ \\
\hline & 11 to 20 & $149(28.4)$ & $36(37.9)$ & $33(34.7)$ & $218(30.5)$ \\
\hline & 21 or more & $324(61.8)$ & $50(52.6)$ & $52(54.7)$ & $426(59.7)$ \\
\hline \multirow[t]{2}{*}{ Gender } & Male & $195(37.1)$ & $30(31.6)$ & $49(51.0)$ & $274(38.2)$ \\
\hline & Female & $331(62.9)$ & $65(68.4)$ & $47(49.0)$ & $443(61.8)$ \\
\hline \multirow[t]{2}{*}{ Marital status } & Single & $303(58.0)$ & $54(56.8)$ & $57(60.0)$ & $414(58.1)$ \\
\hline & Married or living as married & $219(42.0)$ & $41(43.2)$ & $38(40.0)$ & $298(41.9)$ \\
\hline \multirow[t]{2}{*}{ Hispanic/Latino } & Not Hispanic or Latino & $508(97.3)$ & $93(97.9)$ & $90(94.7)$ & $691(97.1)$ \\
\hline & Hispanic or Latino & $14(2.7)$ & $2(2.1)$ & $5(5.3)$ & $21(2.9)$ \\
\hline \multirow[t]{3}{*}{ Race/ethnicity } & White & $260(49.5)$ & $80(84.2)$ & 77 (81.9) & $417(58.4)$ \\
\hline & Black & $201(38.3)$ & $6(6.3)$ & $1(1.1)$ & $208(29.1)$ \\
\hline & Other or multi-racial & $64(12.2)$ & $9(9.5)$ & $16(17.0)$ & $89(12.5)$ \\
\hline \multirow[t]{3}{*}{ Geographic classification } & Rural & $51(9.7)$ & $27(28.4)$ & $11(11.5)$ & $89(12.4)$ \\
\hline & Suburban & $140(26.6)$ & $22(23.2)$ & $26(27.1)$ & $188(26.2)$ \\
\hline & Urban & $336(63.8)$ & $46(48.4)$ & $59(61.5)$ & $441(61.4)$ \\
\hline \multirow[t]{2}{*}{ Education } & GED, HS Degree, or less & $307(59.3)$ & $42(44.2)$ & $40(42.6)$ & $389(55.0)$ \\
\hline & $\begin{array}{l}\text { At least some college, } \\
\text { technical, or trade school }\end{array}$ & $211(40.7)$ & $53(55.8)$ & $54(57.4)$ & $318(45.0)$ \\
\hline \multirow[t]{2}{*}{ Currently employed } & Not employed & $274(52.1)$ & $60(63.2)$ & $51(53.1)$ & $385(53.7)$ \\
\hline & Employed & $252(47.9)$ & $35(36.8)$ & $45(46.9)$ & $332(46.3)$ \\
\hline \multirow[t]{2}{*}{ Living with a disability } & No disability & $387(74.0)$ & $57(60.6)$ & 68 (71.6) & $512(71.9)$ \\
\hline & Disability & $136(26.0)$ & $37(39.4)$ & $27(28.4)$ & $200(28.1)$ \\
\hline \multirow[t]{2}{*}{ Annual household income } & Less than $\$ 20,000$ & $310(61.6)$ & $69(74.2)$ & $51(54.3)$ & $430(62.3)$ \\
\hline & More than $\$ 20,000$ & $193(38.4)$ & $24(25.8)$ & $43(45.7)$ & $260(37.7)$ \\
\hline \multirow[t]{2}{*}{ Dental insurance status } & No dental insurance & $390(74.0)$ & $43(45.3)$ & $50(52.1)$ & $483(67.3)$ \\
\hline & Dental insurance & $137(26.0)$ & $52(54.7)$ & $46(47.9)$ & $235(32.7)$ \\
\hline \multirow[t]{2}{*}{ Last dental cleaning } & Less than 1 year & $65(12.4)$ & $13(13.8)$ & $16(16.8)$ & $94(13.2)$ \\
\hline & Over 1 year ago or never & 459 (87.6) & $81(86.2)$ & 79 (83.2) & $619(86.8)$ \\
\hline \multirow[t]{2}{*}{ Perceived gum disease } & No perceived gum disease & $322(61.1)$ & $56(58.9)$ & $50(52.1)$ & $428(59.6)$ \\
\hline & Perceived gum disease & 205 (38.9) & $39(41.1)$ & $46(47.9)$ & $290(40.4)$ \\
\hline \multirow{2}{*}{$\begin{array}{l}\text { Motivation to see dentist } \\
\text { in next } 6 \text { months }\end{array}$} & Low motivation & $88(16.7)$ & $21(22.1)$ & $25(26.0)$ & $134(18.7)$ \\
\hline & High motivation & $439(83.3)$ & $74(77.9)$ & $71(74.0)$ & $584(81.3)$ \\
\hline \multirow{2}{*}{$\begin{array}{l}\text { Self-efficacy to see dentist } \\
\text { in next } 6 \text { months }\end{array}$} & Low self-efficacy & $168(31.9)$ & $30(31.6)$ & $39(40.6)$ & $237(33.0)$ \\
\hline & High self-efficacy & $359(68.1)$ & $65(68.4)$ & $57(59.4)$ & $481(67.0)$ \\
\hline \multirow[t]{2}{*}{ Dental insurance barrier } & No such barrier & $88(16.8 \%)$ & $42(44.2 \%)$ & $27(28.1 \%)$ & $157(21.9 \%)$ \\
\hline & Dental insurance barrier & $437(83.2 \%)$ & $53(55.8 \%)$ & $69(71.9 \%)$ & $559(78.1 \%)$ \\
\hline \multirow[t]{2}{*}{ Cost barrier } & No such barrier & $74(14.1 \%)$ & $23(24.5 \%)$ & $20(20.8 \%)$ & $117(16.3 \%)$ \\
\hline & Cost barrier & $452(85.9 \%)$ & $71(75.5 \%)$ & $76(79.2 \%)$ & $599(83.7 \%)$ \\
\hline \multirow[t]{2}{*}{ Psychological barriers ${ }^{\mathrm{a}}$} & No such barriers & $247(46.9)$ & $35(36.8)$ & $46(47.9)$ & $328(45.7)$ \\
\hline & Psychological barriers & $280(53.1)$ & $60(63.2)$ & $50(52.1)$ & $390(54.3)$ \\
\hline
\end{tabular}


Table 1 Baseline demographic characteristics of tobacco quitline callers in $\mathrm{OH} 4 \mathrm{~L}$ study by state of residence, $n=718$ (Continued)

\begin{tabular}{|c|c|c|c|c|c|}
\hline & & $\begin{array}{l}\text { Louisiana } \\
N=527 \\
N(\%)\end{array}$ & $\begin{array}{l}\text { Nebraska } \\
N=95 \\
N(\%)\end{array}$ & $\begin{array}{l}\text { Oregon } \\
N=96 \\
N(\%)\end{array}$ & $\begin{array}{l}\text { Overall } \\
N=718 \\
N(\%)\end{array}$ \\
\hline \multirow[t]{2}{*}{ Prioritization barriers ${ }^{a}$} & No such barriers & $128(24.3 \%)$ & 19 (20.0\%) & $29(30.2 \%)$ & $176(24.5 \%)$ \\
\hline & Prioritization barriers & 399 (75.7\%) & $76(80.0 \%)$ & $67(69.8 \%)$ & $542(75.5 \%)$ \\
\hline \multirow[t]{2}{*}{ Access barriers $^{a}$} & No such barriers & $349(66.2 \%)$ & $66(69.5 \%)$ & $66(68.8 \%)$ & $481(67.0 \%)$ \\
\hline & Access barriers & $178(33.8 \%)$ & $29(30.5 \%)$ & $30(31.2 \%)$ & 237 (33.0\%) \\
\hline
\end{tabular}

Missing values: Age (3); Cigarettes per day (4); Gender (1); Marital status (6); Hispanic/Latino (6); Race/ethnicity (4); Education (11); Currently employed (1); Living with a disability (6); Annual household income (28); Last dental cleaning (5)

${ }^{a}$ For our analysis and for ease of interpretation, we grouped similar barriers into the following categories: access (unable to find a dentist, too difficult to get to the clinic), psychological (fear or nervousness, dislike going to the dentist), and prioritization (mean to go but put it off, other health concerns are more important, have no problems with teeth or gums, don't have time, do not think of it)

a dentist in the next six months. Only about one-third of participants reported having dental insurance, $83.7 \%$ cited cost as a barrier to obtaining dental care, and $78.1 \%$ cited a dental insurance barrier. Many participants $(75.5 \%)$ reported one or more prioritization barriers, such as meaning to go but putting it off, or viewing other health concerns as more important. About half of participants (54.3\%) reported psychological barriers, which included fear or nervousness and disliking dental visits. Only about one-third (33.0\%) of participants reported access-related barriers, such as an inability to find a dentist or difficulty getting to the office or clinic.

\section{Association of financial barriers and potential}

\section{demographic confounders with dental care utilization}

When we examined each of our a priori identified financial and demographic factors (dental insurance status, dental insurance barrier, cost barrier, age, gender, and state), we found no association between dental care utilization at 6-month follow-up and either age, gender, or state (Table 2). Consistent with prior research, dental insurance status and perceived dental insurance barriers were associated with receipt of dental care. Participants with dental insurance were more likely to have seen a dentist by 6-month follow-up than those without (RR 1.66 [95\% CI 1.22-2.26]). Participants who reported having a dental insurance barrier to care were less likely to have seen a dentist compared with those who did not report this barrier (RR 0.69 [95\% CI 0.500.97]). Of those who reported cost as a barrier to dental care, $17.5 \%$ had seen a dentist compared with $23.1 \%$ of those who did not report this barrier; however, the cost barrier was not significantly associated with dental care utilization (RR 0.76 [95\% CI 0.52-1.10]).

\section{Associations between baseline characteristics of interest and dental care utilization}

In the single variable adjusted models (Table 3), we found that factors significantly associated with a higher likelihood of dental care utilization at followup included having high levels of motivation to see the dentist (RR 2.86 [95\% CI 1.55-5.29]) and high self-efficacy for seeing

Table 2 Association of a priori identified financial and demographic confounders with dental utilization by 6-month follow-up

\begin{tabular}{|c|c|c|c|c|}
\hline & & Use of Dental Care, \% & Unadjusted RR (95\% Cl) & $p$-value \\
\hline \multirow[t]{3}{*}{ Age } & 18 to 44 & 16.7 & 1 (ref) & 0.661 \\
\hline & 45 to 64 & 19.4 & $1.16(0.84-1.60)$ & \\
\hline & 65 or older & 17.2 & $1.03(0.45-2.37)$ & \\
\hline \multirow[t]{2}{*}{ Gender } & Male & 17.2 & 1 (ref) & 0.496 \\
\hline & Female & 19.2 & $1.12(0.81-1.55)$ & \\
\hline \multirow[t]{3}{*}{ State } & Louisiana & 16.5 & 1 (ref) & 0.090 \\
\hline & Nebraska & 24.2 & $1.47(0.98-2.20)$ & \\
\hline & Oregon & 22.9 & $1.39(0.92-2.10)$ & \\
\hline \multirow[t]{2}{*}{ Dental insurance status } & No dental insurance & 15.1 & 1 (ref) & 0.001 \\
\hline & Dental insurance & 25.1 & $1.66(1.22-2.26)$ & \\
\hline \multirow[t]{2}{*}{ Dental insurance barrier } & No such barrier & 24.2 & 1 (ref) & 0.030 \\
\hline & Dental insurance barrier & 16.8 & $0.69(0.50-0.97)$ & \\
\hline \multirow[t]{2}{*}{ Cost barrier } & No such barrier & 23.1 & 1 (ref) & 0.144 \\
\hline & Cost barrier & 17.5 & $0.76(0.52-1.10)$ & \\
\hline
\end{tabular}


Table 3 Associations between baseline characteristics and dental utilization by 6-month follow-up in single variable adjusted models $^{\mathrm{a}}$

\begin{tabular}{|c|c|c|c|c|}
\hline & & Use of Dental Care, \% & Adjusted RR $(95 \% \mathrm{Cl})^{\mathrm{a}}$ & $p$-value \\
\hline \multirow[t]{2}{*}{ Marital status } & Single & 21.0 & 1 (ref) & \multirow[t]{2}{*}{0.023} \\
\hline & Married or living as married & 14.4 & $0.68(0.48-0.95)$ & \\
\hline \multirow[t]{2}{*}{ Education } & GED, HS Degree, or less & 14.4 & 1 (ref) & \multirow[t]{2}{*}{0.004} \\
\hline & At least some college, technical, or trade school & 23.9 & $1.63(1.17-2.26)$ & \\
\hline \multirow{2}{*}{$\begin{array}{l}\text { Living with a disability (employed \& } \\
\text { unemployed) }\end{array}$} & No disability & 15.2 & 1 (ref) & \multirow[t]{2}{*}{0.005} \\
\hline & Disability & 26.5 & $1.65(1.17-2.32)$ & \\
\hline \multirow[t]{2}{*}{ Last dental cleaning } & Less than 1 year & 36.2 & 1 (ref) & \multirow[t]{2}{*}{$<0.001$} \\
\hline & Over 1 year ago or never & 15.7 & $0.47(0.33-0.67)$ & \\
\hline \multirow[t]{2}{*}{ Perceived gum disease } & No perceived gum disease & 15.8 & 1 (ref) & \multirow[t]{2}{*}{0.066} \\
\hline & Perceived gum disease & 22.1 & $1.35(0.98-1.85)$ & \\
\hline \multirow{2}{*}{$\begin{array}{l}\text { Motivation to see dentist in next } \\
6 \text { months }\end{array}$} & Low motivation & 7.5 & 1 (ref) & \multirow[t]{2}{*}{0.001} \\
\hline & High motivation & 20.9 & $2.86(1.55-5.29)$ & \\
\hline \multirow{2}{*}{$\begin{array}{l}\text { Self-efficacy to see dentist in next } \\
6 \text { months }\end{array}$} & Low self-efficacy & 9.7 & 1 (ref) & \multirow[t]{2}{*}{$<0.001$} \\
\hline & High self-efficacy & 22.7 & $2.26(1.46-3.49)$ & \\
\hline \multirow[t]{3}{*}{ Cigarettes per day } & 10 or fewer & 20 & 1 (ref) & \multirow[t]{3}{*}{0.95} \\
\hline & 11 to 20 & 19.3 & $0.92(0.54-1.58)$ & \\
\hline & 21 or more & 17.8 & $0.93(0.56-1.55)$ & \\
\hline \multirow[t]{2}{*}{ Hispanic/Latino } & Not Hispanic or Latino & 18.7 & 1 (ref) & \multirow[t]{2}{*}{0.35} \\
\hline & Hispanic or Latino & 9.5 & $0.52(0.13-2.07)$ & \\
\hline \multirow[t]{3}{*}{ Race/ethnicity } & White & 20.1 & 1 (ref) & \multirow[t]{3}{*}{0.21} \\
\hline & Black & 16.8 & $0.92(0.62-1.36)$ & \\
\hline & Other or multi-racial & 10.1 & $0.56(0.29-1.07)$ & \\
\hline \multirow[t]{3}{*}{ Geographic classification } & Rural & 21.3 & 1 (ref) & \multirow[t]{3}{*}{0.73} \\
\hline & Suburban & 19.7 & $0.10(0.61-1.6)$ & \\
\hline & Urban & 17.2 & $0.88(0.56-1.39)$ & \\
\hline \multirow[t]{2}{*}{ Currently employed } & Not employed & 19 & 1 (ref) & \multirow[t]{2}{*}{0.72} \\
\hline & Employed & 17.5 & $0.94(0.68-1.30)$ & \\
\hline \multirow[t]{2}{*}{ Annual household income } & Less than $20 \mathrm{~K}$ & 19.5 & 1 (ref) & \multirow[t]{2}{*}{0.25} \\
\hline & Over $20 \mathrm{~K}$ & 16.5 & $0.82(0.58-1.15)$ & \\
\hline \multirow[t]{2}{*}{ Psychological barriers } & No such barriers & 19.2 & 1 (ref) & \multirow[t]{2}{*}{0.31} \\
\hline & Psychological barriers & 17.7 & $0.85(0.61-1.17)$ & \\
\hline \multirow[t]{2}{*}{ Prioritization barriers } & No such barriers & 15.9 & 1 (ref) & 0.61 \\
\hline & Prioritization barriers & 19.2 & $1.11(0.75-1.63)$ & \\
\hline Access barriers & No such barriers & 18.3 & 1 (ref) & 0.99 \\
\hline & Access barriers & 18.6 & $1.00(0.72-1.38)$ & \\
\hline
\end{tabular}

Bolded text indicates associations that are significant at the $p<0.05$ level

${ }^{a}$ A separate model assesses each baseline characteristic of interest one at a time. All models are adjusted for a priori identified financial variables (dental insurance status, dental insurance barrier, cost barrier) and potential confounders (age, gender, and state). All $p$-values are global $p$-values

the dentist (RR 2.26 [95\% CI 1.46-3.49]). Participants with a disability were more likely to see a dentist than those without a disability (RR 1.65 [95\% CI 1.17-2.32]), and those who had at least some college education were more likely to see a dentist compared with those who had a high school education or less (RR 1.63 [95\% CI 1.17-2.26]). Factors significantly associated with a lower likelihood of dental care utilization at followup including being married (RR 0.68 [95\% CI 0.48-0.95]), and not having received a dental cleaning in more than 1 year (RR 0.47 [95\% CI 0.33-0.67]).

All associations that were significant in our single variable models (Table 3) remained significant in our multivariable model (Table 4), though RR estimates were 
Table 4 Associations between baseline characteristics and dental utilization by 6-month follow-up in multivariable adjusted model ${ }^{a}$

\begin{tabular}{|c|c|c|c|c|}
\hline & & $\begin{array}{l}\text { Use of Dental } \\
\text { Care, \% }\end{array}$ & Adjusted RR $(95 \% \mathrm{Cl})^{\mathrm{a}}$ & $p$-value \\
\hline \multirow[t]{3}{*}{ Age } & 18 to 44 & 16.7 & 1 (ref) & 0.96 \\
\hline & 45 to 64 & 19.4 & $0.97(0.69-1.38)$ & \\
\hline & 65 or older & 17.2 & $0.89(0.39-2.03)$ & \\
\hline \multirow[t]{2}{*}{ Gender } & Male & 17.2 & 1 (ref) & 0.83 \\
\hline & Female & 19.2 & $0.97(0.70-1.33)$ & \\
\hline \multirow[t]{3}{*}{ State } & Louisiana & 16.5 & 1 (ref) & 0.45 \\
\hline & Nebraska & 24.2 & $1.14(0.75-1.75)$ & \\
\hline & Oregon & 22.9 & $1.30(0.86-1.96)$ & \\
\hline \multirow[t]{2}{*}{ Dental insurance status } & No dental insurance & 15.1 & 1 (ref) & 0.33 \\
\hline & Dental insurance & 25.1 & $1.20(0.84-1.73)$ & \\
\hline \multirow[t]{2}{*}{ Dental insurance barrier } & No such barrier & 24.2 & 1 (ref) & 0.61 \\
\hline & Dental insurance barrier & 16.8 & $0.90(0.60-1.35)$ & \\
\hline \multirow[t]{2}{*}{ Cost barrier } & No such barrier & 23.1 & 1 (ref) & 0.88 \\
\hline & Cost barrier & 17.5 & $0.97(0.64-1.46)$ & \\
\hline \multirow[t]{2}{*}{ Marital status } & Single & 21.0 & 1 (ref) & 0.02 \\
\hline & Married or living as married & 14.4 & $0.67(0.48-0.94)$ & \\
\hline \multirow[t]{2}{*}{ Education } & GED, HS Degree, or less & 14.4 & 1 (ref) & 0.013 \\
\hline & $\begin{array}{l}\text { At least some college, technical, or } \\
\text { trade school }\end{array}$ & 23.9 & $1.52(1.09-2.12)$ & \\
\hline \multirow[t]{2}{*}{ Living with a disability (employed \& unemployed) } & No disability & 15.2 & 1 (ref) & 0.004 \\
\hline & Disability & 26.5 & $1.63(1.17-2.27)$ & \\
\hline \multirow[t]{2}{*}{ Last dental cleaning } & Less than 1 year & 36.2 & 1 (ref) & $<0.001$ \\
\hline & Over 1 year ago or never & 15.7 & $0.52(0.37-0.72)$ & \\
\hline \multirow[t]{2}{*}{ Perceived gum disease } & No perceived gum disease & 15.8 & 1 (ref) & 0.014 \\
\hline & Perceived gum disease & 22.1 & $1.49(1.09-2.05)$ & \\
\hline \multirow[t]{2}{*}{ Motivation to see dentist in next 6 months } & Low motivation & 7.5 & 1 (ref) & 0.029 \\
\hline & High motivation & 20.9 & $2.16(1.08-4.32)$ & \\
\hline \multirow[t]{2}{*}{ Self-efficacy to see dentist in next 6 months } & Low self-efficacy & 9.7 & 1 (ref) & 0.019 \\
\hline & High self-efficacy & 22.7 & $1.80(1.10-2.93)$ & \\
\hline
\end{tabular}

Bolded text indicates associations that are significant at the $p<0.05$ level

ancludes our a priori identified covariates (cost barrier, dental insurance barrier, having dental insurance [yes/no], gender, age, and state), as well as each variable found to be significant at the $p \leq 0.10$ level in the single variable models (step 1 ). All $p$-values are global $p$-values

somewhat attenuated (RRs 2.16 for motivation, 1.80 for self-efficacy, 0.52 for having a recent dental cleaning, 1.52 for having a higher education level, 0.67 for being married, and 1.63 for living with a disability). The association between perceived gum disease and dental care utilization was near-significant in our single variable models (RR 1.35 [95\% CI 0.98-1.85]) and became significant with multivariable adjustment (RR 1.49 [95\% CI, 1.09-2.05]).

Age, gender, and state all remained unassociated with dental care utilization in the multivariable model. Contrary to our expectations, we found little to no association in the multivariable model between dental care utilization and any financial factors, including dental insurance status (RR 1.20 [95\% CI, 0.84-1.73]), self-reported dental insurance barriers (RR 0.90 [95\% CI, 0.60-1.35]), or self-reported cost barriers (RR 0.97 [95\% CI, 0.64-1.46]).

\section{Post-hoc sensitivity and exploratory analyses}

In sensitivity analyses conducted among individuals whose last dental cleaning was more than 1 year ago $(n=459)$, the single variable adjusted model results mirrored the full cohort analysis, with no change in the significance of any variables nor the direction of association for any significant variables (data not shown). In the multivariable adjusted model, the 
estimated associations were also generally in the same direction and of similar magnitude as found in the broader sample, but due to reduced power, many associations were no longer significant in this subgroup (data not shown).

We also conducted exploratory analyses to further examine our unexpected findings that being single and having a disability were associated with receipt of dental care at follow-up. For this analysis, we compared other baseline characteristics between single vs. married participants as well as between participants with a disability vs. those without. We found that a higher proportion of single participants had completed at least some college $(48.3 \%$ compared to $39.9 \%$ among married participants). We also found that a much higher percentage of individuals living with a disability were unemployed $(92.0 \%$ vs. $38.5 \%$ for those without a disability), and that Nebraska had a higher proportion of participants with a disability (39.4\%) compared with Oregon (28.4\%) and Louisiana $(26.0 \%)$. However, as shown in the multivariable findings, adjusting for education status and state did not change the significance of the association between either disability status or marital status and dental care utilization.

\section{Discussion}

Smokers, particularly those who are low-income or uninsured, are an important target group for promoting dental care utilization. To better inform future efforts to promote dental visits in this high-risk group, we examined baseline factors associated with future dental care among participants in the OH4L trial. We found that significant predictors of future dental care included being single, not reporting dental insurance as a barrier to care, and having dental insurance, high levels of motivation and self-efficacy to visit the dentist, a disability, a higher education level, perceived gum disease, and a dental cleaning within the past year.

Our finding that having dental insurance was associated with a greater likelihood of seeking future dental care was consistent with research from other countries, including studies from Korea [41] and Finland [42] showing an increase in dental visit attendance after a nationwide expansion in public dental insurance coverage. In the U.S., only about $7 \%$ of adults have public dental insurance (provided through Medicaid) and about 60\% have private dental insurance, usually obtained through their employer or on the individual insurance market [43]. About 33\% of U.S. residents - and about $67 \%$ of our study sample - have no dental insurance and therefore bear the cost burden of dental care [43]. In our analysis, we found lower rates of dental care utilization among study participants who reported cost as a barrier to dental care, although this association was not significant. Together, these findings about cost and dental insurance support the notion that financial factors may represent important barriers to accessing dental care, a finding that is consistent with prior research [5, 18-20].

However, we found these financial barriers were no longer significantly associated with the likelihood of seeking dental services after adjusting for other baseline variables. This unexpected finding contrasts with prior research, and suggests financial barriers do little to alter the likelihood of seeking professional dental care after adjusting for other personal characteristics, such as having high levels of motivation and self-efficacy to visit the dentist, perceived gum disease, a more recent dental cleaning, a higher education level, being single, and having a disability.

There are several possible explanations for the discrepancies between prior research and our findings regarding the role of financial factors as barriers to dental care. Most prior studies were conducted among nationally or regionally representative samples of civilian non-institutionalized populations, [18-20] which in some cases were stratified by smoking status [5]. In contrast, our sample consisted exclusively of smokers from Louisiana, Nebraska, and Oregon, most of whom were very low-income. Therefore, the factors that influence dental care utilization among these smokers in our three states might differ from those that influence utilization among a population with a greater diversity of income levels. In addition, most prior studies were cross-sectional analyses that provided the frequencies of participants' self-reported reasons for forgoing needed dental care without adjusting for other personal characteristics, $[5,19,20]$ or assessed the likelihood of different outcomes, such as lack of care for known dental problems [18]. In contrast, our prospective, longitudinal analysis offers insight into the characteristics associated with an increased likelihood of transitioning from being a non-utilizer to a utilizer of dental services, which may involve different influences.

We undertook this analysis to explore factors that might be hindering or facilitating dental visits among high-risk smokers to inform future intervention development. In contrast to our expectations, neither income level nor geographic classification (urban, suburban, rural) were significantly associated with future dental care utilization; however, the former may reflect the lack of economic diversity in our sample. Two-thirds had annual household incomes below $\$ 20,000$, and less than $5 \%$ had annual household incomes over $\$ 60,000$ [15]. Other factors not associated with future dental care utilization included psychological barriers such as fear or nervousness, 
access-related barriers such as difficulty finding a dentist, and prioritization barriers such as viewing other health concerns as more important. We initially expected these factors to play a role in dental utilization, and in fact, the OH4L intervention sought to address these issues through a combination of cognitive behavioral counseling, oral health education, and referrals to local low-cost dental providers. However, our comprehensive, multi-modal intervention had no effect on future dental care [15]. Taken together, the results of our randomized trial and the findings from this secondary analysis suggest income, geographic classification, and perceived barriers may be less important drivers of future dental care among low-income smokers than one's motivation to see a dentist or confidence in one's ability to see the dentist.

Since higher levels of motivation and self-efficacy to visit the dentist were associated with a greater likelihood of future dental care utilization, we recommend future interventions specifically seek to build individuals' motivation and self-efficacy to visit the dentist, independent of their concerns about cost. In addition, our finding that people with perceived gum disease were more likely to see a dental provider suggests future interventions should educate smokers about their gum health and oral disease risk while simultaneously fostering their self-efficacy and motivation to see a dental provider. Based on evidence that gain-framed health risk messaging is more persuasive than loss-framed messaging for oral hygiene behaviors, [44-46] we recommend that such an intervention emphasize the positive benefits of seeing a dentist, as opposed to the health consequences of neglecting this care.

Other significant correlates of future dental care included having a higher education level, being single, and having a disability. While the association between dental care utilization and education aligns with prior research, [22] our findings on marital status and disability status were unexpected. Our exploratory analysis found that single participants generally had higher levels of education than married participants; however, adjusting for education level did not impact the significance of the association between marital status and seeking dental care. In addition, participants with a disability were more likely to report being unemployed than participants without a disability, so it is possible that participants with a disability had more free time to visit the dentist. Nebraska also had a higher proportion of participants with a disability compared with Oregon and Louisiana. However, neither employment status nor state were significantly associated with future dental care use in any of our analyses, suggesting the association between disability status and dental care utilization may be complex and not easily explained by our current analyses or available data.
Overall, our findings highlight the difficulty of increasing dental care utilization among high-risk, low-income smokers and the need for additional research to improve our understanding of how to best support tobacco users in obtaining dental care. For example, future studies could interview smokers about what they would need to schedule, attend, and maintain routine dental visits. Such interviews might uncover factors that were not explored in our present analysis but could play a role in supporting dental visit attendance, such as assistance with appointment scheduling, appointment reminders, or childcare during visits.

\section{Strengths and limitations}

This study has several strengths, including its longitudinal, population-based design that allowed us to follow individuals who transitioned from being non-utilizers to utilizers of dental care. It includes a diverse population of smokers interested in quitting ( $42 \%$ non-White) and a high proportion of very low-income smokers. These are priority populations for intervention because of their high risk of oral disease and low rates of dental care utilization, however, they also face substantial barriers to obtaining dental care. Therefore, understanding the characteristics of participants who transitioned from non-utilizers to utilizers of dental care offers valuable insight for future efforts to facilitate dental care utilization among similar high-risk groups.

This study also has certain limitations. Our sample consisted of a low-income population of smokers from Louisiana, Nebraska, and Oregon who were overdue for dental visits, and our findings may not be generalizable to higher-income smokers, those from other regions or countries, those who visit a dentist regularly, or smokers who have not contacted a quitline for help with tobacco cessation. As a condition of enrollment, participants had to be interested in improving their oral health. As such, the results may not generalize to persons with no interest in improving their oral health. All participant data was based on self-report, and thus subject to bias and misreporting. However, for our main outcome of dental care utilization, our methodology was designed to deter misreporting by requiring a provider name. Since more than $90 \%$ of participants provided this information with an understanding that we may also ask their permission to contact their provider to verify the accuracy of their report, we have great confidence in the veracity of this self-reported data.

\section{Conclusions}

Low-income smokers are at high risk for oral disease and are a priority group for oral health intervention. The findings from our randomized trial underscore the difficulty of increasing dental care utilization among this population, but the current analysis provides useful insight into 
those who are more or less likely to seek dental care. This information can inform the design of future oral health promotion programs either by helping public health officials target those at greatest risk for not seeking dental care (e.g., smokers with lower education levels) or by suggesting potential targets for future behavioral interventions (e.g., perceived disease risk, self-efficacy, motivation). Future research should evaluate whether interventions that target these individuals and address these factors can increase dental care utilization among low-income individuals, particularly smokers.

\section{Abbreviations}

BRFSS: Behavioral Risk Factor Surveillance System; LA: Louisiana; NE: Nebraska; NHANES: National Health and Nutrition Examination Survey; NHIS: National Health Interview Survey; OH4L: OralHealth4Life; OR: Oregon; RR: Relative risk; RUCA: Rural-Urban Commuting Area

\section{Acknowledgements}

We would like to thank study team members at KPWHRI and Optum who made this work possible. At KPWHRI this included Ella Thompson, the Survey Research Program, Ellen Schartz, Mary Shea, Eric Baldwin, Andrew Baer, DT Tran, Deborah King, and Zoe Bermet. At Optum this included Erica Salmon, Mark Campbell, and Mona Deprey. We are also grateful to the Louisiana Campaign for Tobacco-Free Living and the Louisiana Department of Health, Well-Ahead Louisiana; the Oregon Health Authority, Public Health Division, Tobacco Prevention and Education Program; and the Tobacco Free Nebraska, Public Health Division, Nebraska Department of Health of Human Services for supporting this research. Finally, we thank Dr. Dolphine Oda, DDS, MSc; Dr. Johnny Wang, DDS, MPH, MS; and Dr. Helga Ding, DDS, MBA, MHA for consulting on the development of the Oral Health 4 Life intervention.

\section{Funding}

This research was supported by the National Institute of Dental and Craniofacial Research (NIDCR; grant U01DE024462).

\section{Availability of data and materials}

The datasets used and/or analyzed during the current study are available from the corresponding author on reasonable request.

\section{Authors' contributions}

PB led the conceptualization and writing of this manuscript. CK conducted data analyses and wrote sections of the methods. MA and JL oversaw the implementation of the analytic plan. TB oversaw study activities at the Optum Center for Wellbeing Research. SC assisted in intervention development and fidelity oversight. JM conceptualized the study, secured research funding, and oversaw intervention development. All authors interpreted the data, provided input on manuscript drafts, and approved the final manuscript.

\section{Ethics approval and consent to participate}

This research was approved by the Kaiser Permanente Washington Institutional Review Board.

\section{Consent for publication}

$$
\text { Not applicable }
$$

\section{Competing interests}

The authors declare that they have no competing interests.

\section{Publisher's Note}

Springer Nature remains neutral with regard to jurisdictional claims in published maps and institutional affiliations.

\section{Author details}

'Kaiser Permanente Washington Health Research Institute, 1730 Minor Ave, Suite 1600, Seattle, WA 98101, USA. ${ }^{2}$ Department of Biostatistics, University of
Washington, F-600, Health Sciences Building 1705 NE Pacific Street, Seattle, WA 98195, USA. ${ }^{3}$ Optum Center for Wellbeing Research, 999 3rd Ave., Suite 2000, Seattle, Washington 98104, USA. ${ }^{4}$ Betty Irene Moore School of Nursing, University of California-Davis, 2450 48th Street, Suite 2600, Sacramento, CA 95817, USA.

Received: 21 June 2018 Accepted: 18 October 2018

Published online: 01 November 2018

\section{References}

1. U.S. Department of Health and Human Services. Oral Health in America: A Report of Surgeon General. Rockville, MD: U.S. Department of Health and Human Services, National Institute of Dental and Craniofacial Research, National Institutes of Health; 2000.

2. Eke PI, Dye BA, Wei L, Slade GD, Thornton-Evans GO, Borgnakke WS, Taylor GW, Page RC, Beck JD, Genco RJ. Update on prevalence of periodontitis in adults in the United States: NHANES 2009 to 2012. J Periodontol. 2015;86(5): 611-22.

3. SEER Cancer statistics review, 1975-2015. https://seer.cancer.gov/csr/1975_ 2015/.

4. Drury TF, Garcia I, Adesanya M. Socioeconomic disparities in adult oral health in the United States. Ann N Y Acad Sci. 1999:896:322-4.

5. Bloom B, Adams PF, Cohen RA, Simile C. Smoking and Oral health in dentate adults aged 18-64. NCHS data brief, no 85. Hyattsville, MD: Centers for Disease Control and Prevention, National Center for Health Statistics; 2012.

6. Drilea SK, Reid BC, Li CH, Hyman JJ, Manski RJ. Dental visits among smoking and nonsmoking US adults in 2000. Am J Health Behav. 2005;29(5):462-71.

7. Vujicic M, Nasseh K. A decade in dental care utilization among adults and children (2001-2010). Health Serv Res. 2014:49(2):460-80.

8. Institute of Medicine. Advancing Oral health in America. Washington, DC: the National Academies Press; 2011

9. Emanuel AS, Parish A, Logan HL, Dodd VJ, Zheng D, Guo Y. Dental visits mediate the impact of smoking on Oral health. Am J Health Behav. 2018; 42(1):59-68.

10. MCClure JB, Riggs K, St John J, Catz SL. [more] evidence to support oral health promotion services targeted to smokers calling tobacco quitlines in the United States. BMC Public Health. 2013;13:336.

11. Results from the 2016 NAQC Annual Survey of Quitlines [https://www. naquitline.org/page/2016Survey].

12. Disparities in Oral Health [https://www.cdc.gov/oralhealth/oral_health_ disparities/index.htm].

13. McClure JB, Riggs KR, St John J, Cerutti B, Zbikowski S. Understanding oral health promotion needs and opportunities of tobacco quitline callers. Public Health Rep (Washington, DC: 1974). 2012;127(4):401-6.

14. McClure JB. Leveraging the US tobacco quitline infrastructure to promote oral health: feedback from key stakeholders. Prev Med. 2014;60:134-5.

15. McClure JB, Bush T, Anderson ML, Blasi P, Thompson E, Nelson J, Catz SL. Oral health promotion and smoking cessation program delivered via tobacco Quitlines: the Oral health 4 life trial. Am J Public Health. 2018:e1-7.

16. Andersen R. Families' use of health services: a behavioral model of predisposing, enabling and need components [dissertation]. West Lafayette, IN: Purdue University; 1968.

17. Andersen R, Davidson P. Improving access to care in America: individual and contextual indicators. In: Andersen R, Rice T, Kominski E, editors. Changing the US health care system: key issues in Health services, policy, and management. San Francisco: Jossey-Bass; 2001. p. 3-30.

18. Malecki K, Wisk LE, Walsh M, McWilliams C, Eggers S, Olson M. Oral health equity and unmet dental care needs in a population-based sample: findings from the survey of the health of Wisconsin. Am J Public Health. 2015; 105(Suppl 3):S466-74.

19. Wall T, Nasseh K, Vujicic M. Most important barriers to dental care are financial, not supply related. Health policy institute brief. In. Chicago: American Dental Association; 2014.

20. Vujicic M, Buchmueller T, Klein R. Dental care presents the highest level of financial barriers, compared to other types of health care services. Health Aff (Millwood). 2016;35(12):2176-82.

21. Doescher M, Keppel G. Dentist supply, dental care utilization, and Oral health among rural and urban U.S. residents. In: final report \#135. Seattle, WA: WWAMI Rural Health Research Center, University of Washington; 2015. 
22. Reda SF, Reda SM, Thomson WM, Schwendicke F. Inequality in utilization of dental services: a systematic review and meta-analysis. Am J Public Health. 2018;108(2):e1-7.

23. Zhang Y. Racial/ethnic disparity in utilization of general dental care services among US adults: medical expenditure panel survey 2012. J Racial Ethn Health Disparities. 2016;3(4):565-72.

24. Bandura A. Self-efficacy: toward a unifying theory of behavioral change. Psychol Rev. 1977;84(2):191.

25. Hollister MC, Anema MG. Health behavior models and oral health: a review. J Dent Hyg. 2004;78(3):6.

26. Rosenstock IM. The health belief model and preventive health behavior. Health Educ Monogr. 1974;2(4):354-86.

27. Becker MH. The health belief model and personal health behavior. Health Educ Monogr. 1974;2:324-73.

28. Kakudate N, Morita M, Kawanami M. Oral health care-specific self-efficacy assessment predicts patient completion of periodontal treatment: a pilot cohort study. J Periodontol. 2008;79(6):1041-7.

29. Woelber JP, Bienas H, Fabry G, Silbernagel W, Giesler M, Tennert C, Stampf S, Ratka-Kruger P, Hellwig E. Oral hygiene-related self-efficacy as a predictor of oral hygiene behaviour: a prospective cohort study. J Clin Periodontol. 2015;42(2):142-9.

30. McClure JB, Blasi PR, Cook A, Bush T, Fishman P, Nelson J, Anderson ML, Catz SL. Oral health 4 life: design and methods of a semi-pragmatic randomized trial to promote oral health care and smoking abstinence among tobacco quitline callers. Contemp Clin Trials. 2017:57:90-7.

31. Rural-Urban Commuting Area Codes [https://www.ers.usda.gov/dataproducts/rural-urban-commuting-area-codes/].

32. Weeks WB, Kazis LE, Shen Y, Cong Z, Ren XS, Miller D, Lee A, Perlin JB. Differences in health-related quality of life in rural and urban veterans. Am J Public Health. 2004;94(10):1762-7.

33. National Health and Nutrition Examination Survey, 2011-2012 Survey Questionnaires [https://wwwn.cdc.gov/nchs/nhanes/continuousnhanes/ questionnaires.aspx?BeginYear=2011].

34. National Health Interview Survey 2008 [https://www.cdc.gov/nchs/nhis/ data-questionnaires-documentation.htm].

35. Behavioral Risk Factor Surveillance System 2008 [https://www.cdc.gov/brfss/ annual_data/2008/2008_multiple.html].

36. NHIS Data, Questionnaires, and Related Documentation [https://www.cdc. gov/nchs/nhis/data-questionnaires-documentation.htm].

37. Højsgaard S, Halekoh U, Yan J. The R Package geepack for Generalized Estimating Equations. J Stat Softw. 2005;15(2):11.

38. Yan J, Fine J. Estimating equations for association structures. Stat Med. 2004; 23(6):859-74 discussion 875-857,879-880.

39. Yan J: geepack: Yet Another Package for Generalized Estimating Equations. R-News 2002, 2/3:12--14.

40. Nash CM, Vickerman KA, Kellogg ES, Zbikowski SM. Utilization of a webbased vs integrated phone/web cessation program among 140,000 tobacco users: an evaluation across 10 free state quitlines. J Med Internet Res. 2015; 17(2):e36.

41. Park HJ, Lee JH, Park S, Kim TI. Changes in dental care access upon health care benefit expansion to include scaling. J Periodontal Implant Sci. 2016; 46(6):405-14.

42. Linden J, Josefsson K, Widstrom E. Frequency of visits and examinations in the public dental Service in Finland - a retrospective analysis, 2001-2013. BMC Oral Health. 2017;17(1):138.

43. Dental Benefits Coverage in the U.S. [https://www.ada.org/ /media/ADA/ Science\%20and\%20Research/HPI/Files/HPlgraphic_1117_3.pdf?la=en].

44. Brick C, McCully SN, Updegraff JA, Ehret PJ, Areguin MA, Sherman DK. Impact of cultural exposure and message framing on Oral health behavior: exploring the role of message memory. Med Decis Mak. 2016;36(7):834-43.

45. Sherman DK, Updegraff JA, Mann T. Improving oral health behavior: a social psychological approach. J Am Dent Assoc (1939). 2008;139(10):1382-7.

46. Rothman AJ, Salovey P. Shaping perceptions to motivate healthy behavior: the role of message framing. Psychol Bull. 1997;121(1):3-19.

Ready to submit your research? Choose BMC and benefit from:

- fast, convenient online submission

- thorough peer review by experienced researchers in your field

- rapid publication on acceptance

- support for research data, including large and complex data types

- gold Open Access which fosters wider collaboration and increased citations

- maximum visibility for your research: over $100 \mathrm{M}$ website views per year

At BMC, research is always in progress.

Learn more biomedcentral.com/submissions 